\title{
Multi-Objective Optimization of a Fin with Two-Dimensional Heat Transfer Using NSGA-II and ANN
}

M.M Ghanadi Arab ${ }^{1}$, Mohsen Hajabdollahi² and Hassan Hajabdollahi' ${ }^{3 *}$

${ }^{1}$ Department of Electrical Engineering, Islamic Azad University, Science and Recearcher Branch, Tehran, Iran

${ }^{2}$ Department of Computer Engineering, University of Isfahan, Isfahan, Iran

${ }^{3}$ Department of Mechanical Engineering, Vali-e-Asr University of Rafsanjan,Rafsanjan, Iran

\begin{abstract}
Two-dimensional heat transfer in a fin was modeled with acceptable accuracy and optimized. Bezier curve was used to estimate the fin geometry. The finite volume method coupled with the artificial neural network was developed to predict the temperature distribution through the fin with $-1.5 \%$ to $+1 \%$ and $\pm 0.5 \%$ accuracy for fin efficiency and rate of heat transfer, respectively. Locations of four control points in the Bezier curve were considered as design variables. Then, fast and elitist non-dominated sorting genetic algorithm (NSGA-II) was applied to find the maximum fin efficiency and the rate of heat transfer as two objective functions. The results of optimal designs were a set of multiple optimum solutions, called 'Pareto optimal solutions'. The maximum 72 percent for fin efficiency was found with $739 \mathrm{~W}$ as its rate of heat transfer while the maximum rate of heat transfer was $962.3 \mathrm{~W}$ with 57 percent efficiency. In addition, the optimum results of two-dimensional heat transfer were compared with one-dimensional and the average 14.7 percent decreases in fin efficiency and the rate of heat transfer was found that show the deficiency of the one-dimension modeling. In the second case study, the Pareto front was derived for the rate of heat transfer and fin surface area as two objective functions. It was observed that the results of optimum fin configuration in the case of fin efficiency as objective function are the same with the results of fin surface area as objective function.
\end{abstract}

Keywords: Fin; Two-dimensional modeling; Bezier curve; Multiobjective optimization; Artificial neural network

\section{Nomenclature}

$A_{c}:$ Cross-section area $\left(\mathrm{m}^{2}\right)$

$A_{s}:$ Convection heat transfer surface area $\left(\mathrm{m}^{2}\right)$

c: Specific heat $(\mathrm{j} / \mathrm{kg} . \mathrm{K})$

E: Controlled elitism factor

$h$ : Convection heat transfer coefficient $\left(\mathrm{W} / \mathrm{m}^{2} . \mathrm{K}\right)$

$k$ : Fin thermal conductivity (W/m.K)

$L$ : Fin length (m)

$M$ : Number of chromosome

$P_{c}$ : Mutation probability

$P_{m}:$ Crossover probability

Q: Rate of heat transfer (W)

$\dot{q}$ : Internal heat generation $\left(\mathrm{W} / \mathrm{m}^{3}\right)$

$W$ : Fin base length (m)

$T$ : Temperature $(\mathrm{K})$

$t$ : Time (sec)

$Z$ : Length of fin in $\mathrm{z}$ direction $(\mathrm{m})$

\section{Greek abbreviation}

\section{$\eta$ : Fin efficiency (-) \\ $\rho:$ Density $\left(\mathrm{kg} / \mathrm{m}^{3}\right)$}

\section{Subscripts}

b: Fin base conv: Convection heat transfer

$t$ : Total

$\infty$ : Ambient condition

max: Maximum

\section{Introduction}

Fins are extended surfaces to develop heat transfer between the solid and fluid [1]. Fins have variety of applications in several industries such as chilling, air conditioning, heat exchanger, power plant, petrochemical plant, cooling of engines and electrical devices [2]. Fin geometry has a significant effect on the heat transfer and efficiency of the fins. This makes the fin geometry as a main variable to optimize the fin effectively. Cando et al. determined the best geometry of a fin with internal heat generation in the maximum heat transfer operation using calculus of variations [3-4]. They studied curvature of fins with eccentric factor and showed these fins have lower surface at the same heat transfer. Lean et al. studied the circular fins with rectangular profile. They also studied the variation of both conductivity and convection heat transfer coefficient [5]. Azarkish et al. [6] obtained the optimum fin geometry for a single fin and fin array [6,7]. They applied single objective genetic algorithm in a longitudinal fin with one-dimensional heat transfer. Georgiou [8] analyzed pin fin with a fin profile with internal heat

*Corresponding author: Hassan Hajabdollahi, Department of Mechanical Engineering, Vali-e-Asr University of Rafsanjan, Rafsanjan, Iran, E-mail: Hassan.Hajabdollahi@gmail.com

Received December 20, 2012; Accepted January 21, 2013; Published January 26, 2013

Citation: Ghanadi Arab MM, Hajabdollahi M, Hajabdollahi H (2013) MultiObjective Optimization of a Fin with Two-Dimensional Heat Transfer Using NSGAII and ANN. J Appl Mech Eng 2: 117 doi:10.4172/2168-9873.1000117

Copyright: () 2013 Ghanadi Arab MM, et al. This is an open-access article distributed under the terms of the Creative Commons Attribution License, which permits unrestricted use, distribution, and reproduction in any medium, provided the original author and source are credited. 
Citation: Ghanadi Arab MM, Hajabdollahi M, Hajabdollahi H (2013) Multi-Objective Optimization of a Fin with Two-Dimensional Heat Transfer Using NSGA-II and ANN. J Appl Mech Eng 2: 117 doi:10.4172/2168-9873.1000117

generation. Kobus et al. [9]. determined the optimized fin geometry to find minimum fin volume in the certain heat transfer. Molen et al. obtained temperate profile in a pin fin with both convection and radiation heat transfer experimentally and compared the results with a simple heat transfer model [10]. Faberi [11] determined optimum fin geometry using generic algorithm.

In the above works, the one-dimensional heat transfer were modeled and optimized. But in some cases the one-dimensional modeling leads to an error in heat transfer specifications such as temperature distribution and as a result the efficiency and rate of heat transfer. In this work, a two-dimensional heat transfer in a fin was modeled using finite volume method and artificial neural network. Bezier curve was used to determine the fin configuration. The location of four Bezier control points was considered as design variables. Total rate of heat transfer and fin efficiency were considered as two objective functions and multi-objective optimization using NSGA-II technique was applied to find the maximum rate of heat transfer and fin efficiency.

As a summary, the followings are the contribution of this paper into the subject:

- Two-dimensional heat transfer in a fin was modeled and optimized to find the best geometry in both efficiency and rate of heat transfer point of view.

- A closed form equation was derived for objective functions (fin efficiency and rate of heat transfer) versus design variables (decision variables) using artificial neural network with an acceptable accuracy.

- Applying multi-objective optimization for two-dimensional heat transfer in fin with efficiency and rate of heat transfer as two objective functions.

- Comparison of the two and one dimensional heat transfer in the presented case study.

- Considering the rate of heat transfer and fin surface area as another set of objective functions.

\section{The Bezier Curve}

One of the key characteristics of Bezier curves is their shape that is determined by the location of a set of points called control points (Figure 1). Each control point changes the part of the curve nearest to it but has little or no effect on parts of the curve that are farther away. This property allows the designer to make localized changes by moving each control points, without affecting the overall shape of the curve $[12,13]$.

A $n$ order Bezier curve is defined based on $n+1$ points as [12,13]:

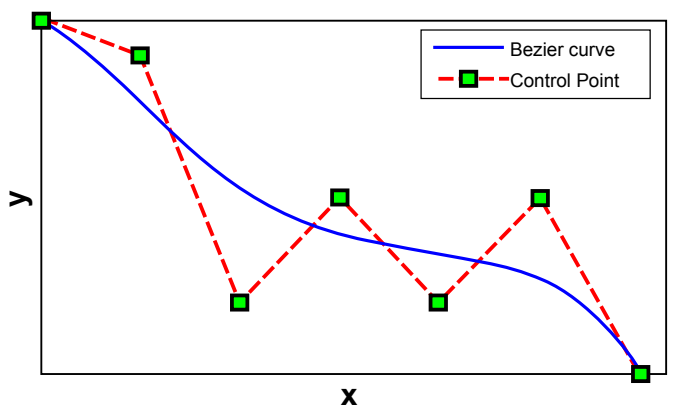

Figure 1: Bezier curve with 7 control points.

$$
C(u)=\sum_{i=0}^{n} B_{i, n}(u) p_{i}, \quad 0 \leq u \leq 1
$$

where pi is the control points, and $B_{i, n}(u)$ is defined as follow:

$$
B_{i, n}(u)=\frac{n !}{i !(n-i) !} u^{i}(1-u)^{n-i}
$$

Where $n$ is the order of Bezier curve and $\mathrm{u}$ is variable in the range of 0 to 1 .

\section{Thermal Modeling}

Three-dimensional heat diffusion equation in the homogeneous medium as well as in the Cartesian coordinate is as follow [1]:

$$
\frac{\partial}{\partial x}\left(k \frac{\partial T}{\partial x}\right)+\frac{\partial}{\partial y}\left(k \frac{\partial T}{\partial y}\right)+\frac{\partial}{\partial z}\left(k \frac{\partial T}{\partial z}\right)+\dot{q}=\rho . c . \frac{\partial T}{\partial t}
$$

where $\dot{q}, \rho$ and $c_{p}$ are internal heat generation, density and specific heat, respectively. In the two-dimensional heat transfer, steady state, constant thermal conductivity and no internal heat generation, the above equation is simplified as follow:

$$
\frac{\partial^{2} T}{\partial x^{2}}+\frac{\partial^{2} T}{\partial y^{2}}=0
$$

The equation (4) is segregated using the finite volume method and applied for the fin to determine the temperature distribution and some other fin specifications such as rate of heat transfer and fin efficiency defined as follow:

$$
\begin{aligned}
& Q=-\left.k A_{c} \frac{d T}{d x}\right|_{x=0} \\
& \eta=\frac{Q}{Q_{\max }}=\frac{Q}{h A_{s, t}\left(T_{b}-T_{\infty}\right)}
\end{aligned}
$$

where $\mathrm{k}$ and $\mathrm{h}$ are conduction and convection heat transfer coefficient respectively, $A_{c}$ and $A_{s, t}$ are the fin cross section area and total convection heat transfer area, respectively. In addition $T_{b}$ is the fin base temperature and $T_{\infty}$ is the ambient temperature. In addition, $Q_{\max }$ is the rate of heat transfer when total convection fin surface has the base temperature? Using the above definition, a fin with the higher efficiency has the better temperature distribution.

\section{Genetic Algorithm (GA) Method}

\section{Multi-objective optimization}

A multi-objective problem consists of optimizing (i.e., minimizing or maximizing) several objectives simultaneously, with a number of inequality or equality constraints. The problem can be formally written as follows:

Find $x=\left(x_{i}\right) \forall i=1,2, \ldots, N_{\text {param }}$ such as

$f_{i}(x)$ is a minimum (respectively maximum) $\forall i=1,2, \ldots, N_{o b j}$

Subject to:

$$
\begin{gathered}
g_{j}(x)=0 \quad \forall j=1,2, \ldots, M, \\
h_{k}(x) \leq 0 \quad \forall k=1,2, \ldots, K,
\end{gathered}
$$

Where is a vector containing the $N_{\text {param }}$ design parameters, 
$\left(f_{i}\right)_{i=1, \ldots ., N o b j}$ the objective functions and $N_{o b j}$ the number of objectives. The objective function returns a vector containing the set of $N_{o b j}$ values associated with the elementary objectives to be optimized simultaneously. The GAs are semi-stochastic methods, based on an analogy with Darwin's laws of natural selection [14]. The first multiobjective GA, called vector evaluated GA (or VEGA), was proposed by Schaffer [15]. An algorithm based on non-dominated sorting was proposed by Srinivas and Deb [16] and called non-dominated sorting genetic-algorithm (NSGA). This was later modified by Deb et al. [17] which eliminated higher computational complexity, lack of elitism and the need for specifying the sharing parameter. This algorithm is called NSGA-II which is coupled with the objective functions developed in this study for optimization.

\section{Non-dominated sorting}

As defined by Deb [18], an individual $X^{(a)}$ is said to constraindominate an individual $X^{(b)}$, if any of the following conditions are true:

(1) $X^{(a)}$ and $X^{(b)}$ are feasible, with

(a) $X^{(a)}$ is no worse than $X^{(b)}$ in all objective, and

(b) $X^{(a)}$ is strictly better than $X^{(b)}$ in at least one objective.

(2) $X^{(a)}$ is feasible while individual $X^{(b)}$ is not.

(3) $X^{(a)}$ and $X^{(b)}$ are both infeasible, but $X^{(a)}$ has a smaller constraint violation.

Here, the constraint violation $\ell(X)$ of an individual $X$ is defined to be equal to the sum of the violated constraint function values [19],

$$
\ell(X)=\sum_{j=1}^{B} \gamma\left(g_{j}(X)\right) g_{j}(X),
$$

Where $\gamma$ is the Heaviside step function. A set of non-dominated individuals is used to form a Pareto-optimal fronts.

\section{Tournament selection}

Each individual competes in exactly two tournaments with randomly selected individuals, a procedure which imitates survival of the fittest in nature.

\section{Controlled elitism sorting}

To preserve diversity, the influence of elitism is controlled by choosing the number of individuals from each subpopulation, according to the geometric distribution [20].

$$
S_{q}=S \frac{1-E}{1-E^{w}} E^{q-1}
$$

To form a parent search population, $P_{t+1}$ ( denote the generation), of size $\mathrm{S}$, where $0<E<1$ and $\mathrm{w}$ is the total number of ranked nondominated.

\section{Crowding distance}

The crowding distance metric proposed by Deb [18] is utilized, where the crowding distance of an individual is the perimeter of the rectangle with its nearest neighbors at diagonally opposite corners. So, if individual $X^{(a)}$ and individual $X^{(b)}$ have same rank, each one has a larger crowding distance is better.

\section{Crossover and mutation}

Uniform crossover and random uniform mutation are employed to obtain the offspring population, $Q_{t+1}$. The integer-based uniform crossover operator takes two distinct parent individuals and interchanges each corresponding binary bits with a probability, $0<p_{c} \leq 1$. Following crossover, the mutation operator changes each of the binary bits with a mutation probability, $0<p_{m}<0.5$.

\section{Historical archive}

The NSGA-II algorithm has been modified to include an archive of the historically non-dominated individuals, $H_{t}$. Archive is used to update the data at each iteration. Figure 2 shows the details about each step of the multi-objective genetic algorithm.

As a summary the following are the steps of NSGA-II with historical archive used in this study for multi-objective optimization:

1. A random population with $M$ chromosome is generated.

2. Each chromosome in the population is decoded using binary decoding.

3. The decoded data are exported to the thermal modeling program and returned back with objective functions (fin efficiency and total rate heat transfer).

4. The population is sorted based on the non-dominated sorting.

5. Then the population is sorted based on the crowding distances.

6. The parent population is formed using the procedure presented in Controlled elitism sorting.

7. A copy of parent is sent to the mating pool and affected by tournament, cross over and mutation operators to forms the new children.

8. The new parent and children are combined and form a new population that is replaced by the older one.

9. This procedure is started again from step 2 until the convergence is met.

\section{Artificial Neural Network}

The feed-forward neural networks are the most popular

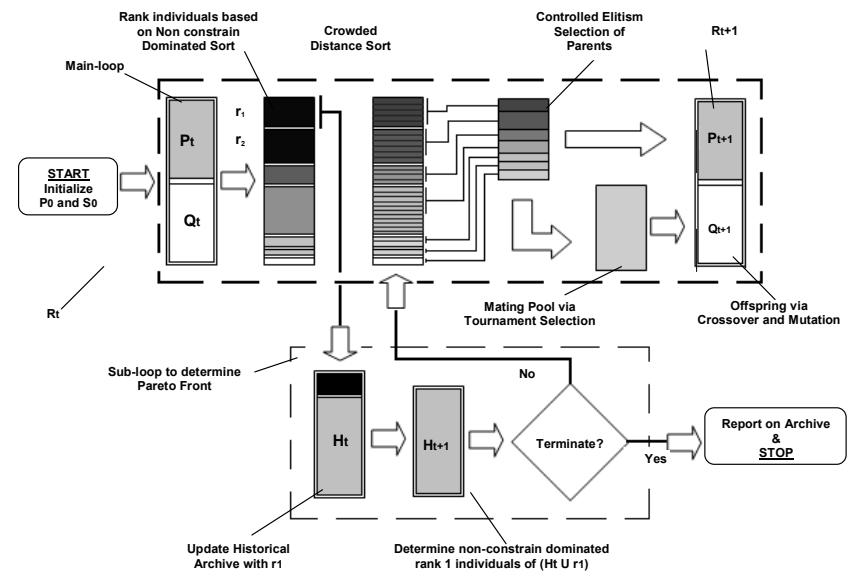

Figure 2: The schematic diagram for the controlled elitist non-dominated sorted multi-objective genetic algorithm with historical archive. 
Citation: Ghanadi Arab MM, Hajabdollahi M, Hajabdollahi H (2013) Multi-Objective Optimization of a Fin with Two-Dimensional Heat Transfer Using NSGA-II and ANN. J Appl Mech Eng 2: 117 doi:10.4172/2168-9873.1000117

Page 4 of 7

architectures due to their structural flexibility, good representational capabilities and availability of a large number of training algorithms [21]. Here the individual element inputs are $I_{1}, I_{2}, I_{R}$ multiplied by weights $w_{11}, w_{12}, \ldots, w_{1 R}$ and the weighted values are fed to the summing junction. The neuron has a bias $b$, which is added with the weighted inputs to form the net input $n$. This sum, $n$, is the argument of the transfer function $F$ :

$$
a=F(n)=F\left(w_{11} I_{1}+w_{12} I_{2}+\ldots+w_{1 R} I_{R}+b\right)
$$

This network consists of neurons arranged in layers in which every neuron is connected to all neurons of the next layer (a fully connected network). We feed the network with input data propagating into the output and the activation function vectors in the network from the input layer to the output layer. This state of the network is called relax state. In the train state of the network the adjustable variables of the networks (weights and biases) are tuned by a proper learning algorithm to minimize the energy function of the network.

\section{Case Study}

In this study, fin efficiency and rate of heat transfer defined in equations (5) and (6) were considered as two objective functions where both of them should be maximized simultaneously. For this purpose a stainless steel fin with heat conductivity $k=20 \mathrm{~W} / \mathrm{m} . \mathrm{K}$ was considered as shown in figure 3 . Ambient temperature was $T_{\infty}=300 \mathrm{~K}$ and convection heat transfer coefficient was assumed to be $h=140$ $W / m^{2} . K$. The fin base temperature was constant at $T b=500 \mathrm{~K}$. The fin dimension in the $x$ and $y$ directions were constant at $L=0.05 \mathrm{~m}$ and $W=0.02 \mathrm{~m}$ respectively. Moreover the heat transfer in the $z$ direction was negligible and fin length in this direction assumed to be $Z=1 \mathrm{~m}$. The design variables were defined as four control points with the fixed $\mathrm{x}$ at $0.01,0.02,0.03$ and $0.04 \mathrm{~m}$ and variable $y$ in the range of $0 \leq y \leq 0.01 \mathrm{~m}$.

\section{Results and Discussion}

\section{Optimization}

Genetic algorithm usually needs at least 100 generation to converge for 4-6 number of design variables [22-24]. Considering the population number $(M=150)$, the finite volume method should be iterated over 15000 times and it takes much computational time. To solve this problem, the artificial neural network was developed to find a closed form correlation between the design variables and the objective functions. For this purpose, the fin geometries were analyzed for 256 states using finite volume method. The first 200 states were used for training the neural network and remain 56 states were hold for verification. Therefore, the neural network by means of a hidden layout with four neurons and an output layout with one neuron, for

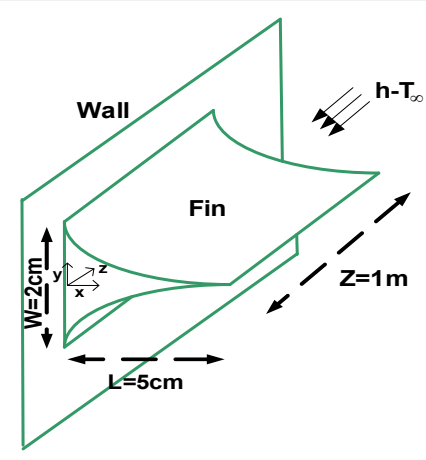

Figure 3: Schematic of the studied fin. four input variables and one output parameter (efficiency or rate of heat transfer) were applied using feed forward algorithm. Tan-sigmoid transfer function $\left(-1+\frac{2}{1+e^{-2 n}}\right)$ was used at the input layout with $\mathrm{n}$ as the transfer function input. Furthermore the linear transfer function $(n)$ was applied at the output layout. A closed form equation for objective functions versus decision variables was derived by training the network as follows:

$$
(Q) \operatorname{or}(\eta)=\sum_{i=1}^{4} w_{i}^{\prime}\left(-1+\frac{2}{1+e^{-2 \sum_{j=1}^{4} w_{i j} x_{j}+b_{i}}}\right)+b^{\prime}
$$

where $b$ and $b^{\prime}$ are the bias value and $w$ and $w^{\prime}$ are weighting function shown separately for each objective functions in tables 1 and 2. The results show that by applying the above proposed correlations (Equation 13), the estimated efficiency and rate of heat transfer, are accurate within $-1.5 \%$ to $+1 \%$ and $\pm 0.5 \%$ respectively (Figures 4 and 5), which are acceptable for engineering problems.

Using the mentioned closed form equation for the objective functions versus design variables, the genetic algorithm optimization was performed for 100 generations, using a search population size of $\mathrm{M}=150$ individuals, crossover probability of $\mathrm{p}_{\mathrm{c}}=0.9$, gene mutation probability of $\mathrm{p}_{\mathrm{m}}=0.035$ and controlled elitism value $\mathrm{E}=0.55$. The results for Pareto-optimal front were shown in figure 6, which clearly reveal

\begin{tabular}{|c|c|c|c|c|c|c|c|}
\hline \multicolumn{4}{|c|}{$w_{i j} \rightarrow i, \downarrow j$} & \multicolumn{4}{|c|}{$w_{i}^{\prime}$} \\
\hline 0.1100 & 1.0182 & 1.9053 & 1.1181 & $0.0 \angle 2301$ & $\begin{array}{r}2049.8 \\
b\end{array}$ & $802.8-c$ & -0005 \\
\hline 0.5924 & 0.3385 & -0.0253 & -0.2108 & & $b_{i}$ & & \\
\hline 0.3276 & 0.8522 & 1.8015 & 4.4131 & -1.2211 & 5.5812 & 2.1783 & 2.7305 \\
\hline 0.3256 & 0.8481 & 1.7960 & 4.4093 & & $\begin{array}{c}b^{\prime} \\
2723\end{array}$ & & \\
\hline
\end{tabular}
the conflict between two objectives, the efficiency and the rate of heat

Table 1: The values of constants in Equation 13 for the estimation of fin efficiency in the presented case study.

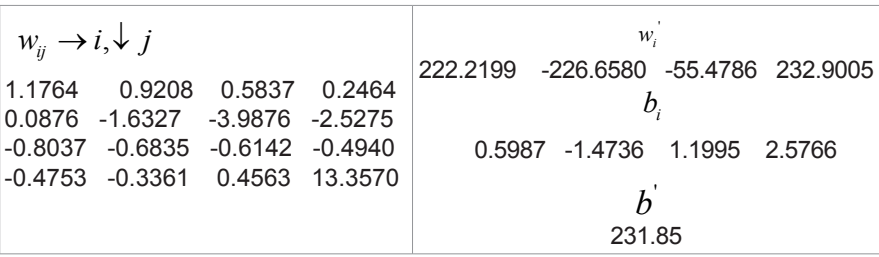

Table 2: The values of constants in Equation 13 for the estimation of rate of heat transfer in the presented case study.

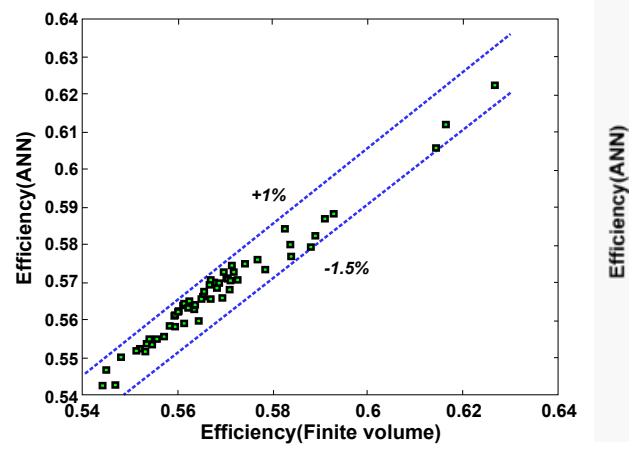

Figure 4: Comparison of fin efficiency using artificial neural network (Eq. 13) and finite volume method in the presented case study. 
Citation: Ghanadi Arab MM, Hajabdollahi M, Hajabdollahi H (2013) Multi-Objective Optimization of a Fin with Two-Dimensional Heat Transfer Using NSGA-II and ANN. J Appl Mech Eng 2: 117 doi:10.4172/2168-9873.1000117

Page 5 of 7

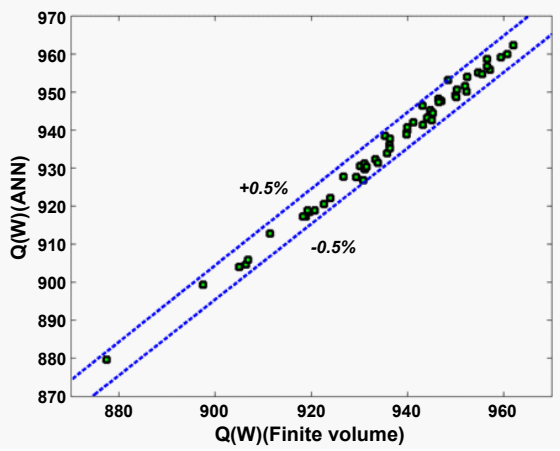

Figure 5: Comparison of rate of heat transfer using artificial neural network (Eq. 13) and finite volume method in the presented case study.

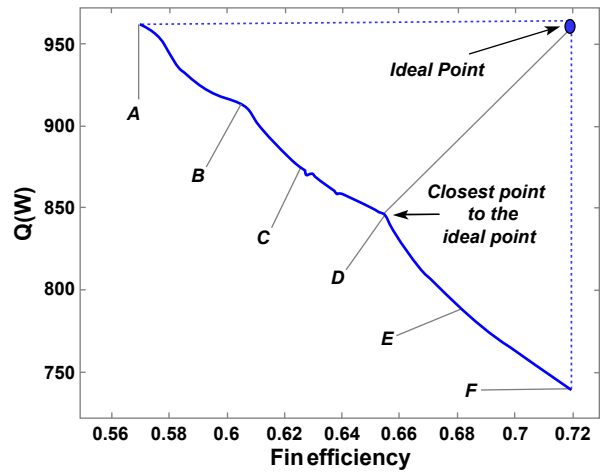

Figure 6: The distribution of Pareto optimal solution (Pareto Front) using NSGA-II when fin efficiency and rate of heat transfer are as two objective functions.

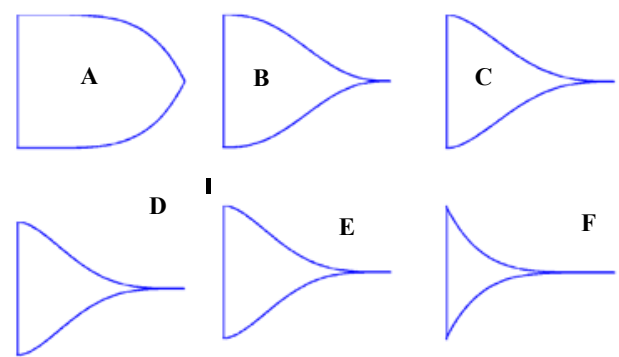

Figure 7: Optimum configuration for six samples of fin shown in Figure 6.

transfer. Any geometrical change that increases the efficiency, leads to a decrease in the rate of heat transfer and vice versa. For example, comparison of points $D$ and $C$ shows relative increasing $4.44 \%$ in fin efficiency and relative decreasing $3.12 \%$ in rate of heat transfer. It is shown in figure 6, that the maximum efficiency exists at design point $F(0.7194)$, while the heat transfer rate is the smallest at this point. On the other hand, the maximum heat transfer occurs at design point $A$ $(962.3 \mathrm{~W})$ with a smallest efficiency value $(0.5708)$ at that point. Design point $F$ is the optimal situation at which, efficiency is a single objective function, while design point $A$ is the optimum condition at which heat transfer rate is a single objective function. The optimum fin geometries for six samples $A-F$ were shown in figure 7 . These configurations were shown dimensionless, where $X=x / L$ and $Y=y / W$. The $A$ configuration was obtained when all control points were at their maximum value
$(0.01 \mathrm{~m})$ while in the $F$ configuration all control points were at their minimum value $(0 \mathrm{~m})$. The results of fin efficiency, rate of heat transfer and fin surface area for optimum design points $A-F$ were reported in table 3 . The corresponding fin surface area versus rate of heat transfer for the Pareto optimal front points was also shown in figure 8. It was observed that the points in this figure are non-dominated. So it can be predicted that fin surface area is conflicted with the rate of heat transfer in the optimum situation. This means that for higher rate of heat transfer, higher fin surface area is required and as a result, higher cost. The Pareto-optimal front with the rate of heat transfer and fin surface area, as two objective functions, was depicted in figure 9 . The results of this Pareto were coincident with the results of figure 8, which clearly confirmed the above fact.

\begin{tabular}{|c|c|c|c|c|c|c|}
\hline & A & B & C & D & E & $\mathrm{F}$ \\
\hline \multicolumn{7}{|l|}{ Two dimensional modeling } \\
\hline Fin efficiency & 0.5708 & 0.6044 & 0.6257 & 0.6535 & 0.6817 & 0.7194 \\
\hline Rate of heat transfer (W) & 962.3 & 913.7 & 874.9 & 847.6 & 787.8 & 739.0 \\
\hline Fin surface area $\left(\mathrm{cm}^{2}\right)$ & 420 & 252 & 192 & 169 & 110 & 85 \\
\hline \multicolumn{7}{|l|}{ One dimensional modeling } \\
\hline Fin efficiency & 0.63564 & 0.68321 & 0.7133 & 0.7497 & 0.79186 & 0.8558 \\
\hline Rate of heat transfer (W) & 1071.5 & 1032.8 & 997.39 & 972.37 & 915.15 & 879.12 \\
\hline Differences (\%) & -11.35 & -13.09 & -14.00 & -14.72 & -16.16 & -18.96 \\
\hline
\end{tabular}

Table 3: Optimum design values for $A$ to $F$ shown in Pareto-optimal fronts

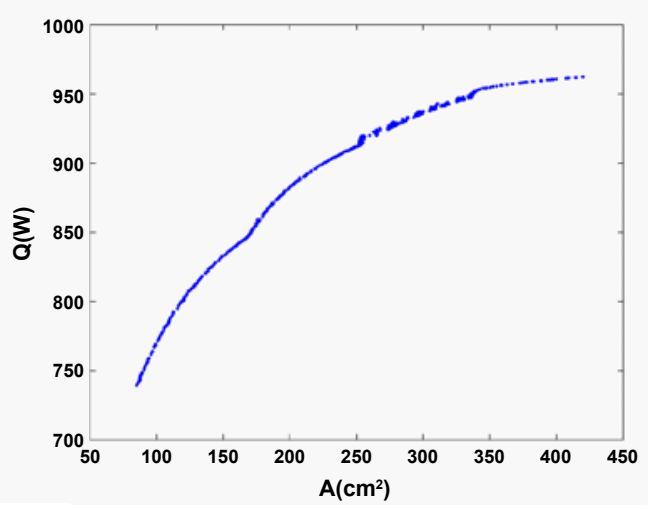

Figure 8: Variation of fin surface area versus rate of heat transfer for optimum design points presented in Figure 6.

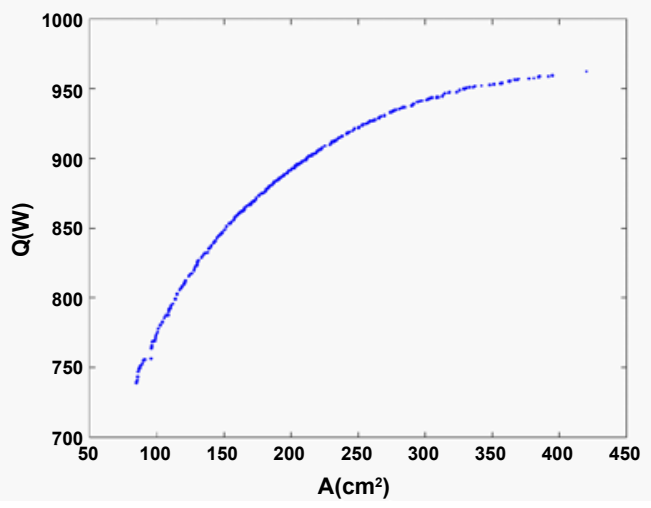

Figure 9: The distribution of Pareto optimal solution using NSGA-II when fin surface area and rate of heat transfer are as two objective functions. 


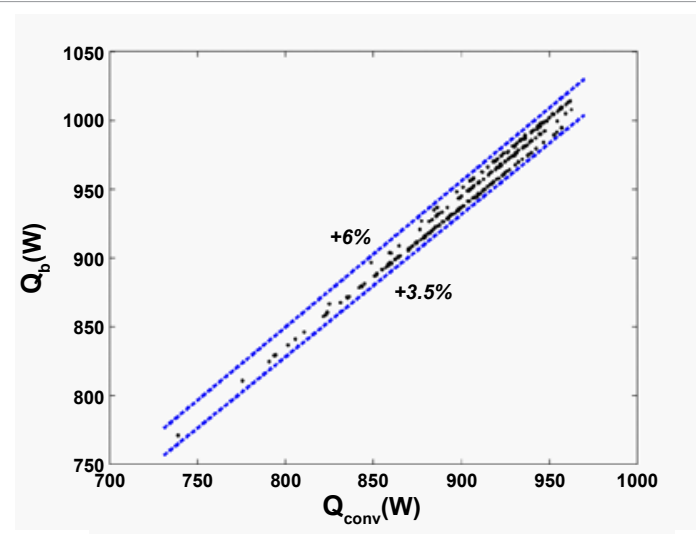

Figure 10: Rate of conduction heat transfer from the fin base versus total convection heat transfer.

\section{Selection of final optimum design}

Each point on the Pareto frontier has the potential of final optimum design. However, selection of final solution among the optimum points existing on the Pareto front needs a process of decision-making. In fact, this process is mostly carried out based on engineering experiences and importance of each objective for decision makers. The process of decision-making is usually performed with the aid of a hypothetical point in figure 6, named as ideal point, that both objectives have their optimal values independent of the other objectives [23].

In this paper, LINMAP method was used to find the final optimum solution in Pareto front [25]. In the LINMAP method, each objective is nondimensionalized using the following relation:

$$
F_{i j}^{n}=\frac{F_{i j}}{\sqrt[2]{\sum_{i=1}^{m}\left(F_{i j}\right)^{2}}}
$$

where $i$ is the index for each point on the Pareto frontier, $j$ is the index for each objective in the objectives space and $m$ denotes the number of points in the Pareto front. After non dimensionalization of two objectives, the distance of each solution on the Pareto frontier from the ideal point obtained. The closest point of Pareto frontier to the ideal point (design point $D$ ) might be considered as a desirable final solution with the 0.6257 fin efficiency and $874.9 \mathrm{~W}$ rate of heat transfer.

\section{Model verification}

Based on the conservation of energy in the steady state and no heat generation, the total conduction heat transfer from the fin base $\left(Q_{b}\right)$ should be the same with the total convection heat transfer $\left(Q_{\text {conv }}\right)$. To investigate the validation of presented finite volume method, the corresponding results of $Q_{b}$ and $Q_{\text {conv }}$ for all optimum points in Pareto front (Figure 6) were shown in figure 10. It revealed the $+3.5 \%$ to $+6 \%$ differences between conduction heat transfer and convection heat transfer which is acceptable for engineering problems.

\section{Comparison of two and one dimensional heat transfer modeling}

To have a good insight into the presented two-dimensional heat transfer modeling, the results of fin efficiency and rate of heat transfer in one-dimensional heat transfer for six typical points A-F were also listed in table 3. The results show the decrement of fin efficiency and consequently the rate of heat transfer in two-dimensional modeling than the one-dimensional. The maximum $18.96 \%$ error was found at design point $\mathrm{F}$ and it means the one-dimensional modeling fail further at design point with higher fin efficiency. Moreover the average $14.7 \%$ difference was found for six mentioned points that verify the need for two-dimensional heat transfer modeling for fin.

\section{Conclusions}

Multi-objective shape optimization of fin with two-dimensional heat transfer was modeled and optimized. Bezier curve was used to estimate the fin geometry and finite volume method was applied to obtain the temperature distribution. Locations of four control points in the Bezier curve were considered as design variables. In the presented optimization problem, efficiency and rate of heat transfer were two objective functions (both of them were maximized). A set of Pareto optimal front points were shown. The results revealed the level of conflict between the two objectives. The optimum results of two-dimensional heat transfer modeling were compared with onedimensional and the average $14.7 \%$ heat transfer decreases was found in the two-dimensional. Moreover the reduction was found further at design point with higher fin efficiency and it shows the need for two-dimension heat transfer modeling. In addition the optimization problem was developed when fin surface area and rate of heat transfer were as two objective functions. The optimum results obtained with fin surface area and rate of heat transfer as two objectives were same as the results when the fin efficiency and rate of heat transfer were as two objective functions. It can be concluded that the fin efficiency and fin surface area are proportional in the optimum situation. Finally, an error analysis based on the energy conservation was carried out and results show the acceptable precision.

\section{References}

1. Bergman TL, Lavine AS, Dewitt DP, Incropera FP (1985) Introduction to Heat Transfer. Wiley, New York, USA.

2. Kern DQ, Kraus AD (1972) Extended Surface Heat Transfer. McGraw-Hill, USA

3. Kundu B, Das PK (2005) Optimum Profile of Thin Fins with Volumetric Heat Generation: A Unified Approach. J Heat Trans 127: 945.

4. Kundu B, Das PK (1999) Performance Analysis and Optimization of Eccentric Annular Disk Fins. J Heat Trans 121:128.

5. YU LT, Chen CK (1999) Optimization of circular fins with variable thermal variables. J Franklin Ins 336: 77-95

6. Azarkish H, Sarvari SMH, Behzadmehr A (2010) Optimum geometry design of a longitudinal fin with volumetric heat generation under the influences of natural convection and radiation. Energ Conver Manage 51: 1938-1946.

7. Azarkish H, Sarvari SMH, Behzadmehr A (2010) Optimum design of a longitudinal fin array with convection and radiation heat transfer using a genetic algorithm. Int J Therm Sci 49: 2222-2229.

8. Georgiou EN (1998) Analysis and Optimization of Convective Pin Fins with Trapezoidal Profile having Internal Heat Generation Density. J Franklin Ins 335: 179-197.

9. Kobus CJ, Cavanaugh RB (2006) A Theoretical Investigation Into the Optima Longitudinal Profile of a Horizontal Pin Fin of Least Material Under the Influence of Pure Forced and Pure Natural Convection With a Diameter-Variable Convective Heat Transfer Coefficient. J Heat Trans 128: 843-846.

10. Mueller Jr. DW, Abu-Mulaweh $\mathrm{HI}$ (2006) Prediction of the Temperature in a Long Horizontal Fin Rod Cooled by Natural Convection and Radiation. Appl Therm Eng 26: 1662-1668.

11. Fabbri G (1997) A genetic algorithm for fin profile optimization. Int J Heat Mass Tran 40: 2165-2172.

12. Piegl L, Tiller W (1997) The NURBS book. Springer-Verlag, Berlin, Germany.

13. Gerald CF, Wheatley PO (1999) Applied Numerical Analysis. VV books, USA. 
Citation: Ghanadi Arab MM, Hajabdollahi M, Hajabdollahi H (2013) Multi-Objective Optimization of a Fin with Two-Dimensional Heat Transfer Using NSGA-II and ANN. J Appl Mech Eng 2: 117 doi:10.4172/2168-9873.1000117

Page 7 of 7

14. Goldberg DE (1989) Genetic Algorithms in Search, Optimization and Machine Learning. Addison-Wesley, USA.

15. Schaffer JD (1985) Multiple objective optimization with vector evaluated genetic algorithms. Proceedings of the international conference on genetic algorithm, USA.

16. Srinivas N, Deb K (1994) Multiobjective optimization using nondominated sorting in genetic algorithms. J Evol Comput 2: 221-248.

17. Deb K, Pratap A, Agarwal S, Meyarivan T (2002) A fast and elitist multiobjective genetic algorithm: NSGA-II. IEEE Trans Evol Comput 6: 182-197.

18. Deb K (2001) Multi-objective optimization using evolutionary algorithms. (1 edn), John Wiley and Sons Ltd, USA.

19. Deb K (2000) An efficient constraint handling method for genetic algorithms. Comput Method Appl Mech Eng 186: 311-338.

20. Deb K, Goel T (2001) Controlled elitist non-dominated sorting genetic algorithms for better convergence. Proceedings of the first international conference on evolutionary multi-criterion optimization.
21. Haykin S (1999) Neural networks: a comprehensive foundation. ( $2^{\text {nd }}$ edn) Prentice-Hall, USA.

22. Ahmadi P, Hajabdollahi H, Dincer I (2010) Cost and Entropy Generation Minimization of a Cross-Flow Plate Fin Heat Exchanger Using Multi-Objective Genetic Algorithm. J Heat Trans 133: 21801.

23. Hajabdollahi H, Ahmadi P, Dincer I (2010) An Exergy-Based Multi-Objective Optimization Of A Heat Recovery Steam Generator (HRSG) In A Combined Cycle Power Plant (CCPP) Using Evolutionary Algorithm. Int J Green Energ 8: 44-64.

24. Hajabdollahi H, Ahmadi P, Dincer I (2011) Modeling and Multi-Objective Optimization of Plain Fin and Tube Heat Exchanger Using Evolutionary Algorithm. Int J Thermo physics Heat Trans.

25. Sayyaadi H, Mehrabipour R (2012) Efficiency enhancement of a gas turbine cycle using an optimized tubular recuperative heat exchanger. Energy 38: 362375 